\title{
In Vivo Trafficking and Targeting of N-Cadherin to Nascent Presynaptic Terminals
}

\author{
James D. Jontes, Michelle R. Emond, and Stephen J Smith \\ Department of Molecular and Cellular Physiology, Stanford University School of Medicine, Stanford, California 94305
}

\begin{abstract}
$\mathrm{N}$-cadherin is a prominent component of developing and mature synapses, yet very little is known about its trafficking within neurons. To investigate $\mathrm{N}$-cadherin dynamics in developing axons, we used in vivo two-photon time-lapse microscopy of N-cadherin - green fluorescent protein (Ncad-GFP), which was expressed in Rohon-Beard neurons of the embryonic zebrafish spinal cord. Ncad-GFP was present as either stable accumulations or highly mobile transport packets. The mobile transport packets were of two types: tubulovesicular structures that moved preferentially in the anterograde direction and discrete-punctate structures that exhibited bidirectional movement. Stable puncta of Ncad-GFP accumulated in the wake of the growth cone with a time course. Colocalization of Ncad-GFP puncta with synaptic markers suggests that $\mathrm{N}$-cadherin is a very early component of nascent synapses. Expression of deletion mutants revealed a potential role of the extracellular domain in appropriate $\mathrm{N}$-cadherin trafficking and targeting. These results are the first to characterize the trafficking of a synaptic cell-adhesion molecule in developing axons in vivo. In addition, we have begun to investigate the cell biology of $\mathrm{N}$-cadherin trafficking and targeting in the context of an intact vertebrate embryo.
\end{abstract}

Key words: adhesion; imaging; spinal; synaptogenesis; Rohon-Beard; in vivo

\section{Introduction}

Protein and membrane trafficking are vitally important for the development and function of neurons (Hirokawa, 1998). The developing axon, in particular, relies heavily on the delivery of membranes and proteins from the cell body. Synaptogenesis can occur simultaneously with axon elongation, leaving behind a string of en passant synapses in the wake of the growth cone (Hatada et al., 1999; Jontes et al., 2000). In this regard, trafficking of synaptic components to the distal extent of the growing axon is a prerequisite for ongoing synaptogenesis, because of the continual depletion of presynaptic components.

The primary vehicle for trafficking of protein and membrane components in axons has been shown to be tubulovesicular structures and large membranous organelles (Tsukita and Ishikawa, 1980). Time-lapse microscopy, in conjunction with green fluorescent protein (GFP) labeling of synaptic vesicle proteins, has been used to characterize the dynamics of these tubulovesicular structures in cultured neurons (Nakata et al., 1998; Ahmari et al., 2000; Shapira et al., 2003). These diverse tubulovesicular and pleiomorphic membrane compartments cotransport an array of

\footnotetext{
Received Dec. 6, 2003; revised Aug. 26, 2004; accepted Aug. 31, 2004.

This work was supported by a National Institutes of Health Grant (S.J.S.) and a gift from the Vincent Coates Foundation. J.D.J. was supported by a Career Award in the Biomedical Sciences from the Burroughs Wellcome Fund. M.R.E. was supported by a predoctoral National Research Service Award from the National Institute of Neurological Disorders and Stroke. We thank Hitoshi Okamoto for the islet-1 enhancer, Judith Eisen for the zebrafish embryonic CDNA library, and Reinhard Köster and Scott Fraser for UAS and Gal4VP16 containing plasmids. We also thank W. James Nelson and members of the Smith laboratory for helpful discussions and for critical comments on this manuscript.

Correspondence should be addressed to James D. Jontes, B100 Beckman Center, Stanford University School of Medicine, Stanford, CA 94305-5345.E-mail: jontes@stanford.edu.

DOI:10.1523/JNEUROSCI.5399-04.2004

Copyright $\odot 2004$ Society for Neuroscience $\quad$ 0270-6474/04/249027-08\$15.00/0
}

synaptic components (Kraszewski et al., 1995; Okada et al., 1995; Nakata et al., 1998; Ahmari et al., 2000; Zhai et al., 2001). At present, little is known about the dynamics of transport packets in developing axons in vivo, and most studies have focused on the accumulation of synaptic vesicles and vesicle precursor membranes.

$\mathrm{N}$-cadherin is a $\mathrm{Ca}^{2+}$-dependent homophilic, cell adhesion molecule (Takeichi, 1990; Kemler, 1992). The cytoplasmic domain interacts with $\beta$-catenin, and the cadherin- $\beta$-catenin dimer mediates an interaction with the actin cytoskeleton through $\alpha$-catenin (Nagafuchi and Takeichi, 1989; Ozawa et al., 1989). A number of studies have indicated that N-cadherin is an early and potentially important component of developing synapses. N-cadherin is localized to synapses in cultured hippocampal neurons (Benson and Tanaka, 1998), hippocampal and cerebellar slices (Fannon and Colman, 1996), developing somatosensory cortex (Huntley and Benson, 1999), and in biochemically purified synapses (Phillips et al., 2001). Evidence suggests that cadherin-based adherens junctions are present at the synaptic active zone, as well as perisynaptically, encircling the active zone (Fannon and Colman, 1996; Uchida et al., 1996; Phillips et al., 2001). Although a specific role for N-cadherin has not been definitively demonstrated, evidence has suggested that it could play a role in presynaptic assembly (Bamji et al., 2003), spine morphogenesis (Togashi et al., 2002), activity-dependent plasticity (Tang et al., 1998; Bozdagi et al., 2000; Tanaka et al., 2000), and synaptic specificity (Fannon and Colman, 1996).

To characterize the dynamics of $\mathrm{N}$-cadherin trafficking and delivery to nascent synapses, we have used in vivo two-photon time-lapse microscopy of N-cadherin-GFP (Ncad-GFP) in Rohon-Beard neurons of the embryonic zebrafish spinal cord. Our data reveal that Ncad-GFP is extensively trafficked in the growing 
axons of Rohon-Beard cells in two distinct types of transport packet. In addition, we observe that Ncad-GFP accumulates in stable puncta in the wake of the migrating growth cone, at sites that are most likely nascent synapses. Finally, we show that deletion of the extracellular domain, but not the cytoplasmic domain, prevents the punctate accumulation of $\mathrm{N}$-cadherin.

\section{Materials and Methods}

Cloning of $\mathrm{N}$-cadherin and vesicle-associated membrane protein. To obtain members of the zebrafish cadherin superfamily, degenerate PCR primers (Yamamoto et al., 1998) were used to generate PCR products representing cadherin ectodomain fragments. These were subcloned using the Zero Blunt II TOPO cloning vector (Invitrogen, Carlsbad, CA). One of these clones was highly homologous to mammalian N-cadherin. This fragment was digoxigenin (DIG)-labeled using the Roche PCR DIG labeling kit, and was used to screen a $15-19 \mathrm{hr}$ post-fertilization embryonic zebrafish cDNA library (courtesy of Dr. J. Eisen, University of Oregon) by standard methods. A clone containing an open reading frame of 2682 nucleotides was obtained, encoding for a full-length $\mathrm{N}$-cadherin protein of 894 amino acids. The sequence is identical to that of AF418565 (Lele et al., 2002), which was deposited in GenBank during the course of this work.

A search of the zebrafish expressed sequence tag database for homologs of vesicle-associated membrane protein (VAMP)-synaptobrevin revealed a number of partial overlapping sequences, which could be assembled into a full-length coding sequence. PCR primers were designed against this sequence and used to generate a full-length VAMP clone by RT-PCR, using an initial template of mRNA prepared from zebrafish embryos.

Generation of fusion proteins. Fusion of GFP to full-length N-cadherin, VAMP, and N-cadherin deletion mutants was performed using PCR to place KpnI sites at the $5^{\prime}$ end of the coding regions and AgeI sites at the $3^{\prime}$ end. In all cases, the KpnI-AgeI fragments were subcloned into the Clontech (Palo Alto, CA) GFP-N1 vector to generate fusion proteins containing GFP on the $\mathrm{C}$ termini. For expression in Rohon-Beard cells, the GFP-N1 vector was modified by removing the cytomegalovirus (CMV) promoter of the vector and replacing it with the $14 \times \mathrm{UAS} / \mathrm{E} 1 \mathrm{~b}$ promoter (Köster and Fraser, 2001). Expression of these transgenes was mediated by driving expression of Gal4VP16 (Köster and Fraser, 2001) with the SSICP enhancer-promoter fragment from the islet-1 gene (Higashijima et al., 2000).

DNA was pressure-injected into 1-4 cell stage embryos, using 25-30 $\mu \mathrm{g} / \mathrm{ml}$ each of both the SSICP:Gal4VP16 and UAS:GFP-fusion plasmids. On the next day, healthy embryos were screened for expression of GFP or GFP fusion proteins in Rohon-Beard cells.

Imaging. Selected embryos were anesthetized in $0.003 \%$ tricaine, embedded in $1.5-2 \%$ agarose, and imaged on a custom-made two-photon microscope, designed and assembled by S.J.S. (Jontes et al., 2000; Niell et al., 2004), with the Ti:Sapphire laser (Coherent, Santa Clara, CA) tuned to $920 \mathrm{~nm}$ and using a Zeiss (Oberkochen, Germany) 0.9 numerical aperture (NA) water-immersion objective. For the collection of timelapse data, 20 sections were collected at $1 \mu \mathrm{m}$ steps at time intervals of 2 min for $2-8 \mathrm{hr}$, and at $0.19 \mu \mathrm{m} / \mathrm{pixel}$.

For cyan fluorescent protein (CFP)-yellow fluorescent protein (YFP) colocalization, embryos were mounted on a two-photon microscope, and fluorescence was detected using a dichromatic mirror 505DCXR, an HQ480/40X barrier filter to collect CFP fluorescence, and an D535/30M barrier filter to collect YFP fluorescence (Chroma Technology Corporation, Rockingham, VT).

Analysis. For analysis of time-lapse data, maximum intensity projections were made from median filtered sections for each time point. Analysis was performed on otherwise unmanipulated images. The formation of stable puncta was analyzed by measuring the time interval between the first trace of a stable punctum and the time when the leading edge of the growth cone first reached that position. Although somewhat arbitrary, this provides an approximate measure of time to formation of a stable punctum relative to initial contact between putative presynaptic and postsynaptic sites.

Hippocampal cell culture, transfection, and immunostaining. Primary neuron cultures from rat hippocampus were prepared. Briefly, hip- pocampi were dissected from embryonic day 18 Sprague Dawley rats, dissociated, and plated onto coverslips coated with poly-L-lysine. Cultures were kept at $37^{\circ} \mathrm{C}$ in $5 \% \mathrm{CO}_{2}$. The neuronal cultures ( $8 \mathrm{~d}$ in vitro) were transfected with CMV:Ncad-GFP using a modified calcium phosphate transfection protocol (Xia et al., 1996). After $24 \mathrm{hr}$, the cultures were fixed in methanol at $-20^{\circ} \mathrm{C}$ for $10 \mathrm{~min}$, blocked in $10 \%$ normal goat serum (NGS) in PBS for $1 \mathrm{hr}$ at $37^{\circ} \mathrm{C}$, incubated in primary antibody in PBS with 3\% NGS for $2 \mathrm{hr}$ at room temperature [mouse anti-rat postsynaptic density-95 (PSD-95), 1:100; Affinity BioReagents, Golden, CO; or mouse anti-rat N-cadherin, 1:100, BD Transduction Labs, San Diego, CA), and finally secondary antibody (goat anti-mouse Cy5, 1:100; Jackson ImmunoResearch, West Grove, PA) for $1 \mathrm{hr}$ at room temperature. All procedures were approved by the Institutional Animal Care and Use Committee of Stanford University. Imaging was done with a scanning laser confocal microscope designed by S.J.S. using a Zeiss $40 \times / 1.3 \mathrm{NA}$ Fluar objective. Ten sections were taken $0.5 \mu \mathrm{m}$ apart. Ncad-GFP and PSD-95-Cy5 puncta were considered to be colocalized if they overlapped by at least $50 \%$. Images were collected and analyzed with custom software (N. E. Ziv; Technion, Haifa, Israel).

Whole-mount immunocytochemistry. Ncad-GFP-expressing embryos were fixed in BT fix $\left(0.1 \mathrm{M} \mathrm{PO}_{4}\right.$ buffer, $0.15 \mathrm{~mm} \mathrm{CaCl}_{2}, 4 \%$ sucrose $)$ overnight at $4{ }^{\circ} \mathrm{C}$. The embryos were washed five times in wash buffer $(1 \%$ DMSO, $0.8 \%$ Triton X-100, and $1 \mathrm{mg} / \mathrm{ml} \mathrm{BSA}$ ) for $5^{\prime}$ and blocked for 30 min in wash buffer supplemented with $2 \%$ normal goat serum. The embryos were labeled with a 1:200 dilution of anti-SV2 (developed by K. M. Buckley and obtained from the Developmental Studies Hybridoma Bank, University of Iowa) for $5 \mathrm{hr}$ at $4^{\circ} \mathrm{C}$. The embryos were washed with several changes of wash buffer over $2 \mathrm{hr}$ and incubated in goat antimouse Cy5 (Jackson ImmunoResearch) at a 1:200 dilution for $2 \mathrm{hr}$. Embryos were washed extensively and imaged on a confocal microscope using a Zeiss NA $0.7520 \times$ dry objective.

\section{Results}

\section{Expression of N-cadherin-GFP in Rohon-Beard neurons}

Zebrafish $\mathrm{N}$-cadherin was cloned from an embryonic zebrafish cDNA library and fused at its $\mathrm{C}$ terminus to GFP. A similar fusion of the mouse $\mathrm{N}$-cadherin gene with GFP has been made and was shown to be functional and correctly targeted in fibroblasts (Mary et al., 2002). Efficient gene expression can be obtained in Rohon-Beard neurons using an enhancer element from the islet-1 gene (Higashijima et al., 2000). We used this enhancer element to drive expression of GFP (Fig. 1a) and Ncad-GFP (Fig. $1 b$ ) in developing Rohon-Beard neurons. Soluble GFP efficiently loaded Rohon-Beard neurons, exhibiting a continuous distribution throughout the cells, including thin filopodia. In contrast, Ncad-GFP exhibited perinuclear labeling, as well as a discontinuous localization on both the peripheral axonal arbor and the longitudinal axons, in addition to a faint labeling of the cell body plasma membrane.

\section{$\mathrm{N}$-cadherin-GFP is localized to synapses}

Although N-cadherin has been shown to be present at developing synapses, we wanted to verify that our Ncad-GFP fusion had a proper intracellular localization and that it was targeted to synapses. First, we transfected cultured hippocampal neurons with the zebrafish Ncad-GFP and performed immunocytochemistry with antibodies against PSD-95 (Fig. $2 a-d$ ). Cultured hippocampal neurons transfected with zebrafish Ncad-GFP exhibited a punctate distribution similar to that previously shown for $\mathrm{N}$-cadherin by antibody labeling (Benson and Tanaka, 1998). Of these Ncad-GFP puncta, $74 \%(n=212)$ were found to colocalize with PSD-95 (Fig. $2 a-d$ ), showing that the zebrafish Ncad-GFP was properly targeted to synapses, even in rat neurons. As discussed below, the remaining $26 \%$ of puncta likely represent $\mathrm{N}$-cadherin transport packets. In addition, we used immunostaining with antibodies to the synaptic vesicle protein, SV2, to 

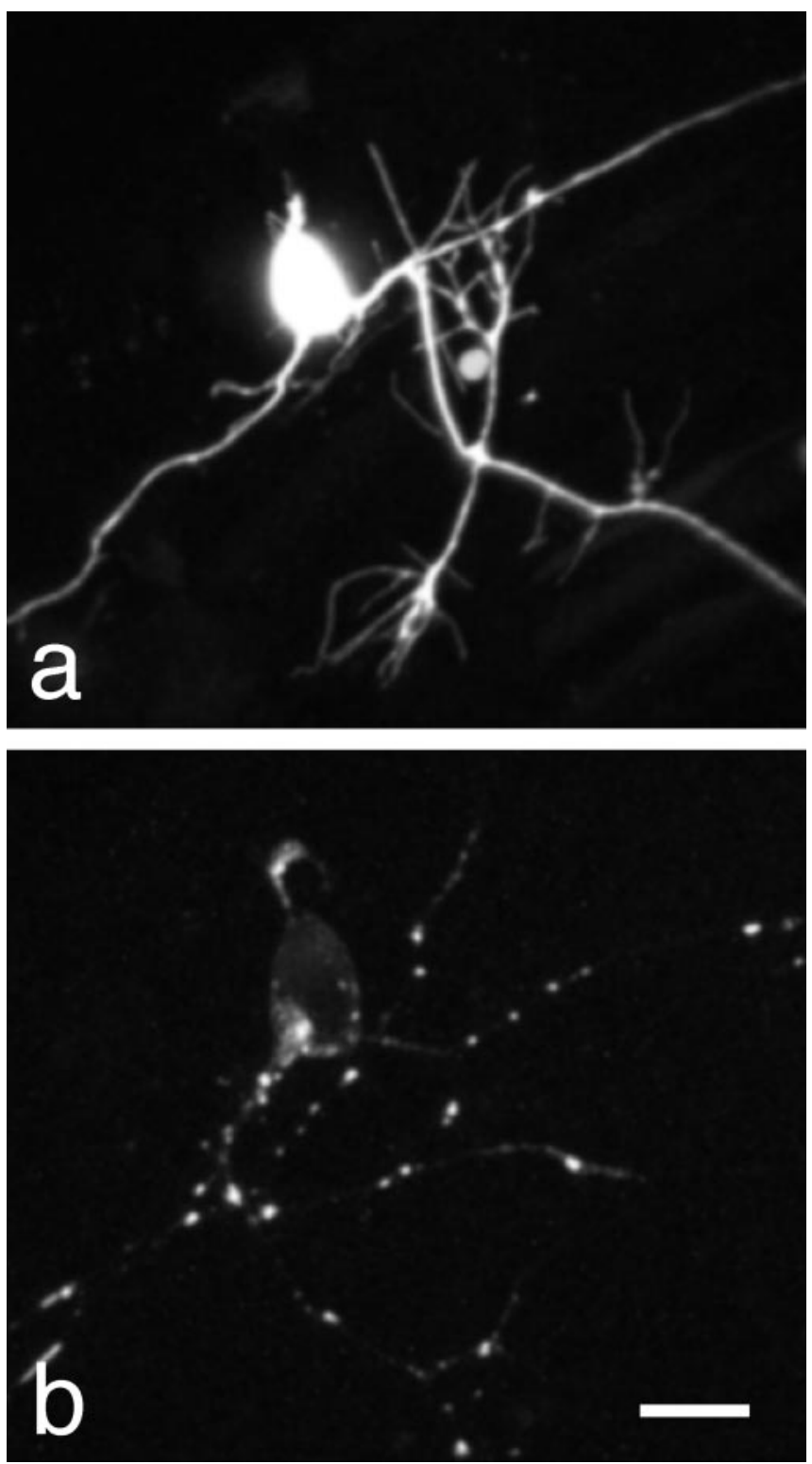

Figure 1. Expression of N-cadherin-GFP in Rohon-Beard neurons. a, A maximum intensity projection of a two-photon image stack showing expression of soluble GFP in a Rohon-Beard neuron of a $\sim 24$-hr-old zebrafish embryo. GFP fluorescence is both continuous and bright within the neuronal processes, effectively labeling even thin filopodia. B, A maximum intensity projection of a two-photon image stack showing expression of an Ncad-GFP fusion protein in a Rohon-Beard neurons of a $\sim 30 \mathrm{hr}$ old zebrafish embryo. In contrast to soluble GFP, Ncad-GFP shows highly punctate staining within the longitudinal axons, as well as the peripheral sensory arbor. In addition, there is a faint labeling of the cell body plasma membrane, but very little background labeling in the axons. Scale bar, $8 \mu \mathrm{m}$.

show synaptic localization of Ncad-GFP in zebrafish neurons (Fig. $2 e-g$ ). In isolated Ncad-GFP-expressing axons, large Ncad-GFP puncta were found to be colocalized with SV2, suggesting synaptic localization. VAMP-synaptobrevin is an integral membrane protein present in synaptic vesicles and is often used as a synaptic marker. Coexpression of Ncad-YFP with VAMPCFP also supports the synaptic localization of $\mathrm{N}$-cadherin. When the same Rohon-Beard cell expressed both VAMP-CFP and Ncad-YFP, the large Ncad-YFP puncta colocalized with accumulations of VAMP-CFP (Fig. $2 h-j$ ).
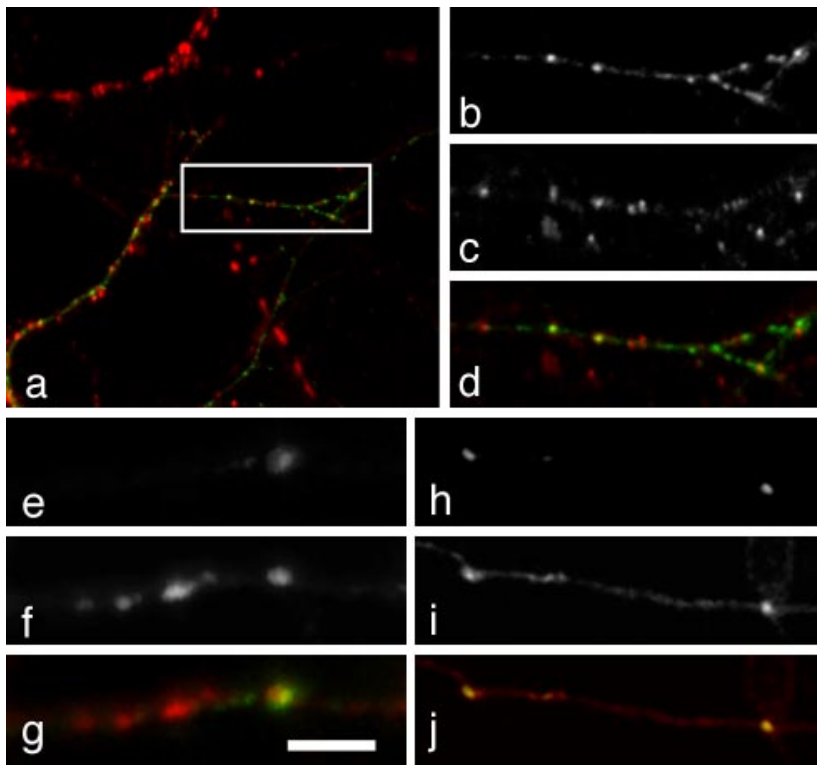

Figure 2. Synaptic localization of zebrafish Ncad-GFP in cultured rat hippocampal neurons. a, Image of a cultured hippocampal neuron transfected with Ncad-GFP (green) and immunolabeled with an antibody against rat PSD-95 (red). When expressed in hippocampal neurons, zebrafish Ncad-GFP exhibits a punctate distribution as is seen in zebrafish embryos in vivo and by previous immunolabeling studies in vitro. $b$, A higher magnification of the boxed area in $a$, showing the punctate distribution of the Ncad-GFP. c, The same area showing staining for rat PSD-95. $d$, The same area showing antibody labeling for PSD-95. $h$, An overlay showing extensive colocalization of Ncad-GFP (green) with PSD-95 (red). Quantitation of the data reveals that there is $74 \%$ colocalization ( $n=212 \mathrm{Ncad}-$ GFP puncta) between the fusion protein and the synaptic marker.e, A confocal image of an axon from a lateral-line ganglion neuron expressing Ncad-GFP, showing dim, diffuse labeling along the axon, except for a large punctum. $f$, The same segment of axon immunostained with an antibody against the synaptic vesicle protein, SV2. $g$, An overlay of the Ncad-GFP and SV2 showing that the Ncad-GFP colocalizes with a large SV2 punctum. Scale bar: (in $g$ ) $e-g, 5 \mu \mathrm{m} ; h-j, 11 \mu \mathrm{m}$. $h$, A two-photon image of a segment of an Ncad-YFP-labeled Rohon-Beard axon in a live embryo. $i$, The same area labeled with VAMP-CFP. $j$, Overlay of the Ncad-YFP and VAMP-CFP signals, showing colocalization of the $\mathrm{N}$-cadherin with accumulations of VAMP.

\section{Transport packets}

Two-photon time-lapse imaging of Ncad-GFP expressed in developing Rohon-Beard axons revealed extensive trafficking of Ncad-GFP in discrete-punctate structures (Fig. 3) (supplemental material, available at www.jneurosci.org). Along the length of labeled axons, Ncad-GFP only faintly and diffusely labeled the axon shaft. The majority of the Ncad-GFP was present in puncta that varied in fluorescent intensity. Most of the puncta were highly mobile and likely represent intracellular transport organelles, moving rapidly in both anterograde and retrograde directions. In addition to the discrete transport packets, we observed elongated fluorescent structures that traveled along axons as a unit (Fig. 4) (supplemental material, available at www.jneurosci.org). These tubulovesicular transport structures were similar to those observed by both electron microscopy and light microscopy of cultured DRG neurons (Tsukita and Ishikawa, 1980; Nakata et al., 1998). The tubulovesicular transport organelles were sometimes difficult to see and were probably detectable because of the high signal-to-noise ratio provided by the very low background of Ncad-GFP in the axons. The small, discrete puncta were far more numerous than the tubulovesicular packets and exhibited a range of fluorescence intensities, suggesting that they represented variably sized clusters of vesicles or pleiomorphic transport organelles. The tubulovesicular structures were rare compared with the discrete-punctate structures and moved preferentially in the anterograde direction $(85 \%$; 33 of 39 

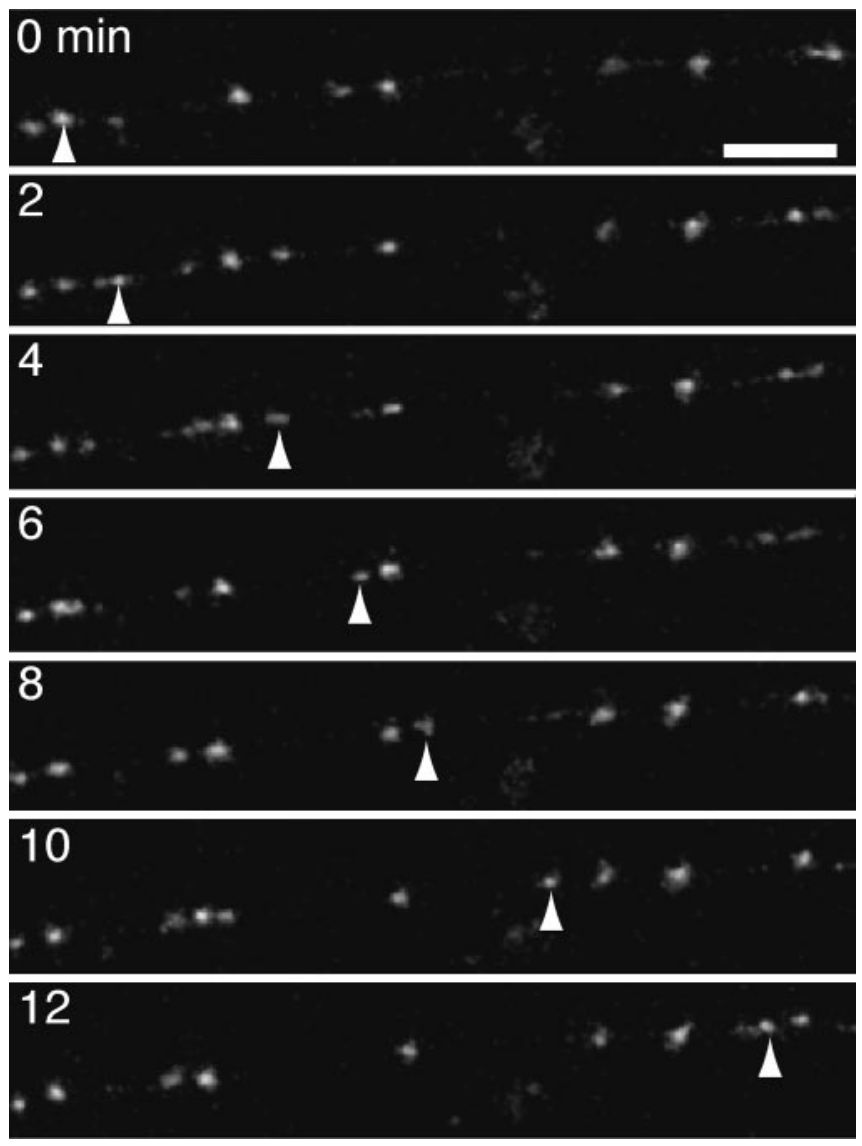

Figure 3. Ncad-GFP labels discrete-punctate transport organelles. Shown are images taken from an in vivo time-lapse sequence, revealing the movement of discrete-punctate transport organelles in a Rohon-Beard axon. Axons exhibit both stable and highly dynamic fluorescent puncta, with very little background staining of the axons. The discrete-punctate transport organelles move in either the anterograde or retrograde directions and reach velocities of up to 34 $\mu \mathrm{m} / \mathrm{min}$. The arrowheads follow one such transport organelle. Scale bar, $10 \mu \mathrm{m}$.

clearly identifiable tubulovesicular transport organelles), indicating that they may be responsible for delivery of cargo to the advancing growth cone.

\section{Formation of stable $\mathrm{N}$-cadherin puncta}

In addition to the ongoing traffic of Ncad-GFP containing transport packets, the developing axons also contained stable NcadGFP puncta. Stable puncta are defined as those that remained immobile from the time of their formation until the end of the time-lapse experiment. Because $\mathrm{N}$-cadherin is a well known component of synapses, and our data suggest that our GFP fusion is correctly targeted to synapses, we analyzed the formation of stable puncta in greater detail. We observed 15 instances of the formation of astableNcad-GFP punctumalong a growing RohonBeard axon. In each case, the first trace of the punctum occurred within a short time after the passage of the growth cone (Fig. 5a) (supplemental material, available at www.jneurosci.org). In general, almost all of the puncta were stabilized by $60 \mathrm{~min}$ after the passage of the leading edge of the growth cone, with the average time to detection being $32 \mathrm{~min}$ (Fig. $5 b)(n=15)$.

Because our above data support the idea that stable NcadGFP puncta label synapses, we wanted to compare the time course of Ncad-GFP accumulation to that of a GFP-tagged zebrafish VAMP-GFP. In young axons, the diffuse background of VAMP-GFP masked the trafficking of small transport packets,
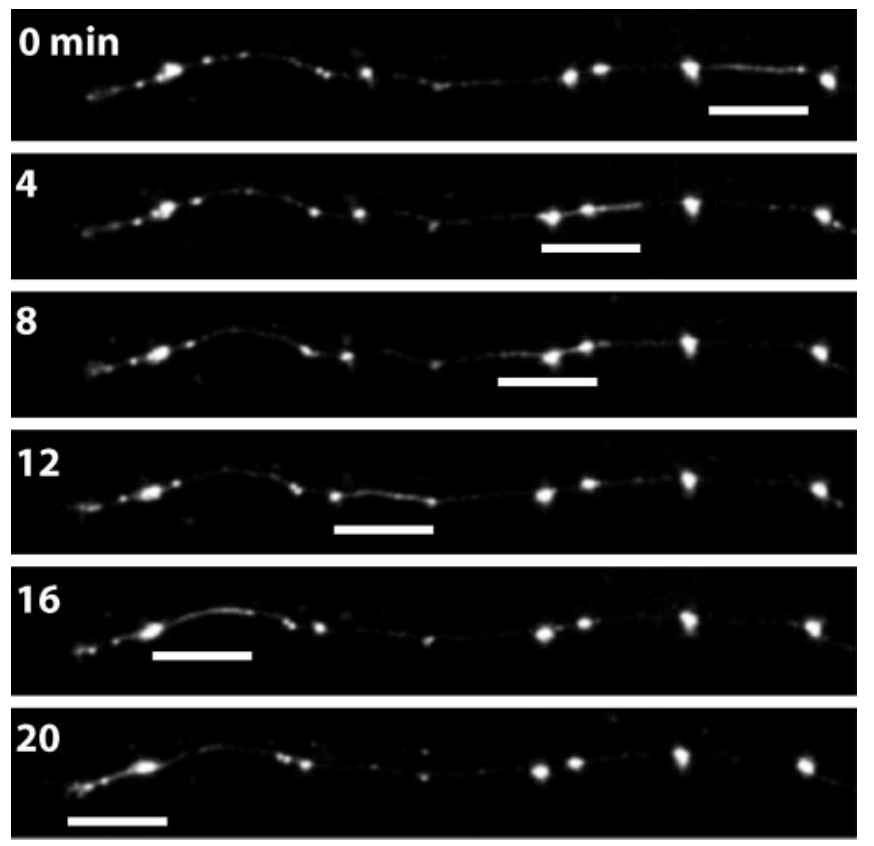

Figure 4. Ncad-GFP labels tubulovesicular transport organelles. Shown are images taken from an in vivo time-lapse sequence, revealing the anterograde transport of a tubulovesicular organelle. In contrast to the discrete-punctate transport organelles, the tubulovesicular structures stain an extended portion of the axon, in some cases up to $\sim 20 \mu \mathrm{m}$. This extended accumulation of fluorescence moves en masse within the axon, predominantly in the anterograde direction. Scale bars, $13 \mu \mathrm{m}$.

characteristic of Ncad-GFP. However, large accumulations of VAMP-GFP, left in the wake of the migrating growth cone, were evident in two-photon time-lapse series of labeled Rohon-Beard neurons (Fig. 6a) (supplemental material, available at www. jneurosci.org). The time course of stable punctum formation is shown in Figure 6b. Analysis of the formation of stable VAMPGFP puncta revealed that the average lag between growth cone passage and punctum formation was $29 \mathrm{~min}$ and that most puncta had been formed within $\sim 60 \mathrm{~min}$, a time course very similar to that determined above for Ncad-GFP.

\section{Localization of $\mathrm{N}$-cadherin deletion mutants}

We wanted to further investigate the trafficking of $\mathrm{N}$-cadherin in Rohon-Beard axons and characterize the determinants of its targeting to nascent synapses. To do this we made two N-cadherin deletion mutants and fused them to GFP:Ncad $\Delta \mathrm{CP}-\mathrm{GFP}$, which has a deletion of the entire cytoplasmic domain, and Ncad $\Delta \mathrm{EC}-$ GFP, which has a deletion of cadherin ectodomains 1-4. As was the case for the full-length Ncad-GFP (Fig. 7a), Ncad $\Delta$ CP-GFP exhibits a highly punctate distribution, with relatively little membrane labeling (Fig. 7b) (supplemental material, available at www. jneurosci.org). In contrast, Ncad $\Delta \mathrm{EC}-\mathrm{GFP}$ diffusely labeled the plasma membrane and did not accumulate as stable discreet puncta (Fig. 7c) (supplemental material, available at www.jneurosci. org). Some trafficking of the Ncad $\Delta \mathrm{EC}-\mathrm{GFP}$ in the axons was visible above the background of the plasma membrane labeling, but this did not result in stable accumulation of fluorescent puncta.

Because deletion of the cytoplasmic domain does not prevent the accumulation of stable Ncad-GFP puncta, we wanted to determine the time course of this accumulation. Two-photon timelapse analysis of Ncad $\Delta \mathrm{CP}$ - GFP-expressing Rohon-Beard neurons revealed dynamic behavior that was qualitatively similar to that exhibited in Ncad-GFP-expressing cells. There was exten- 

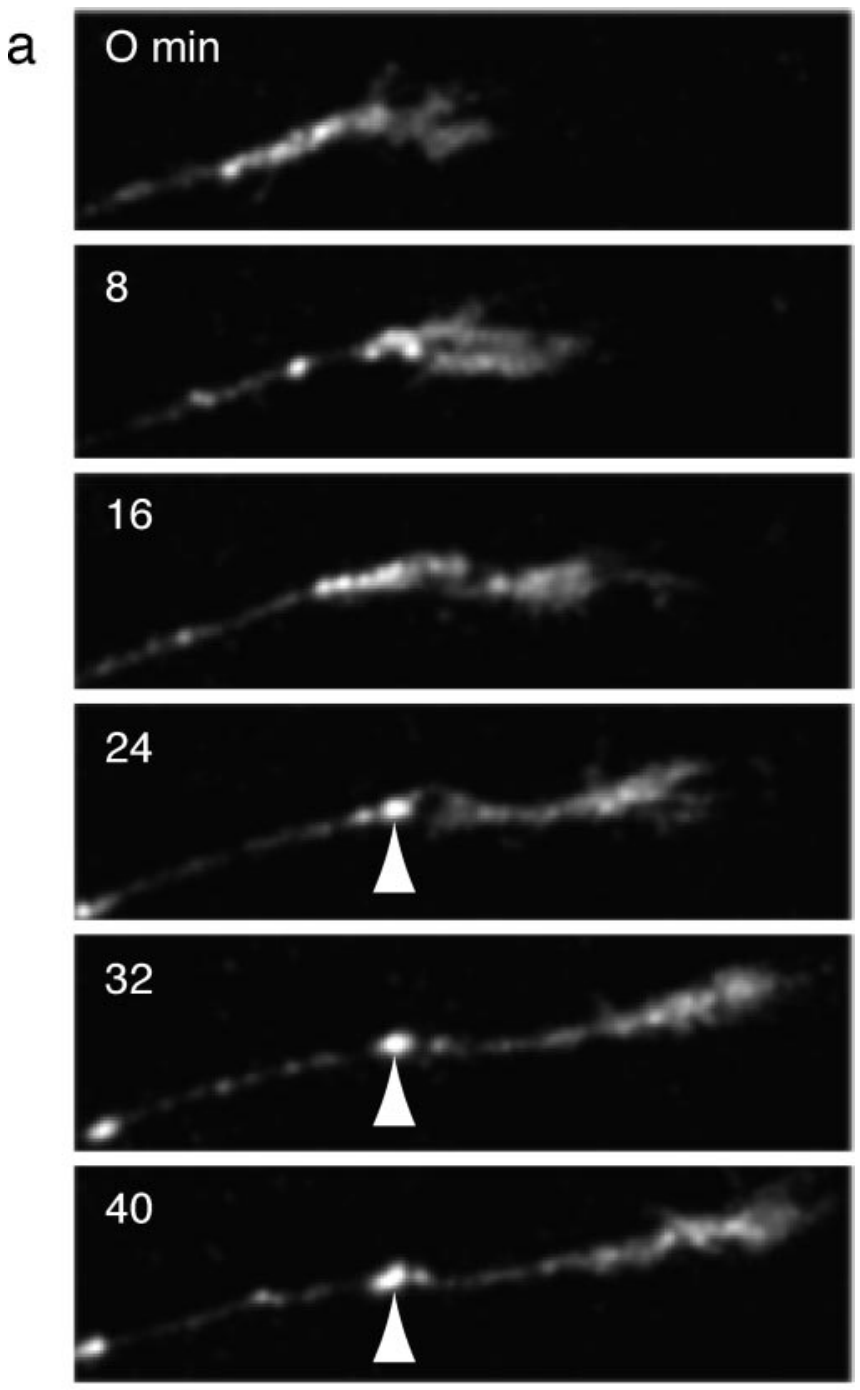

b

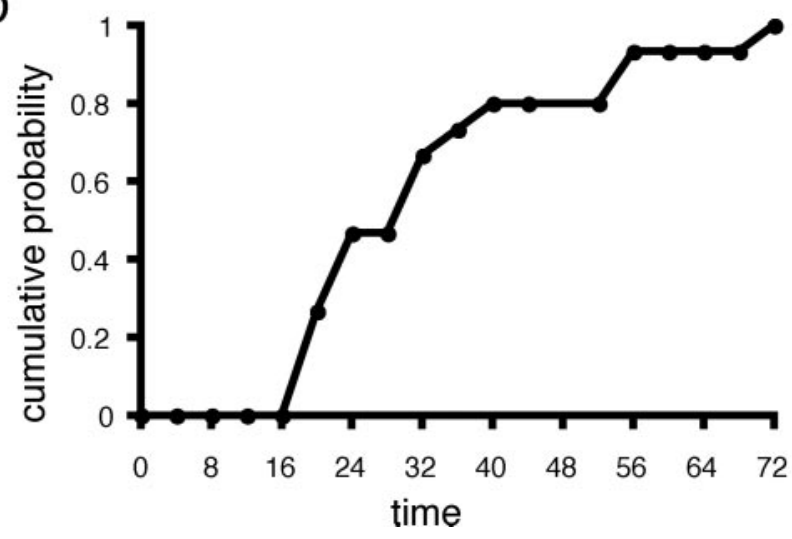

Figure 5. Formation of a stable Ncad-GFP punctum. $a$, In addition to the mobile fluorescent puncta present in the developing Rohon-Beard axons, there are also stable puncta. The stable accumulations (arrowhead), as shown in this time-lapse sequence, form soon after the departure of the migrating axonal growth cone. Scale bar, $10 \mu \mathrm{m}$. $b$, Cumulative probability of stable punctum formation. The formation of multiple stable puncta were characterized relative to the axonal growth cone. The fraction of puncta formed was plotted as a function of time after initial growth cone arrival. The plot shows that nearly all stable Ncad-GFP accumulations form within $1 \mathrm{hr}$ after arrival of the growth cone, with an average time to formation of $32 \mathrm{~min}$. a
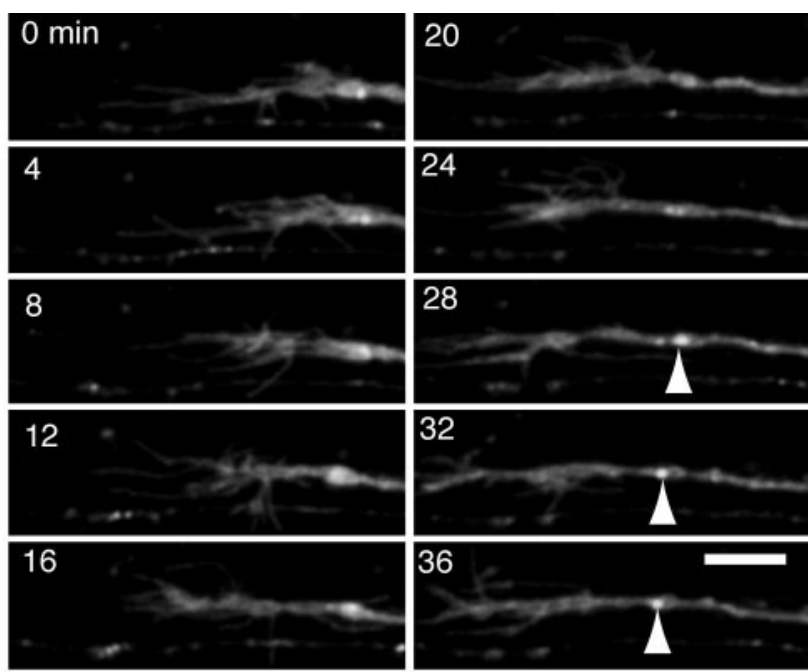

b

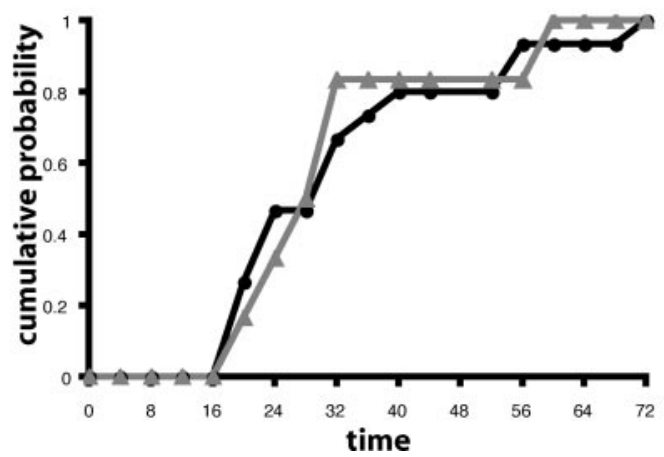

Figure 6. Formation of a stable VAMP-GFP punctum. a, VAMP-GFP is a well characterized marker of synaptic vesicle clusters. Shown is a time-lapse sequence revealing the formation of a stable accumulation of zebrafish VAMP-GFP (arrowhead) in the wake of a migrating growth cone, similar to what we have shown for Ncad-GFP. b, A plot of cumulative probability for VAMP-GFP accumulation, similar to that shown in Figure $5 b$. The time course of VAMP-GFP accumulation (gray trace, gray triangles) was essentially the same as for Ncad-GFP (black trace, black circles), indicating that they accumulate with similar kinetics.

sive trafficking of both discrete-punctate and tubulovesicular transport packets, and Ncad $\Delta$ CP-GFP accumulated in stable puncta in the wake of the migrating growth cone (Fig. 7d). Surprisingly, Ncad $\Delta \mathrm{CP}-\mathrm{GFP}$ accumulated in stable fluorescent puncta with slightly faster kinetics than that of either Ncad-GFP or VAMP-GFP, with a mean time to punctum formation of 25 min after growth cone $(n=12)$. Despite the small difference in the kinetics of accumulation, the mean interpunctum spacing along the axons was identical for Ncad-GFP $(25.9 \mu \mathrm{m} ; n=27)$ and Ncad $\Delta$ CP-GFP $(25.9 \mu \mathrm{m} ; n=24)$, supporting the idea that they marked the same structures. These numbers are similar to that observed for VAMP-GFP $(24.3 \mu \mathrm{m} ; n=16)$.

\section{Discussion}

Adhesion is believed to be an essential early step in initiating synapse assembly in the CNS. A large number of studies have demonstrated a synaptic localization of N-cadherin in the developing and mature CNS (Beesley et al., 1995; Fannon and Colman, 1996; Uchida et al., 1996; Benson and Tanaka, 1998; Huntley and Benson, 1999; Phillips et al., 2001), making it one of the most prominent synaptic cell-adhesion molecules. Despite this, very little is known about the dynamics of $\mathrm{N}$-cadherin in developing neurons, how it is delivered to synapses or its timing of delivery relative to synaptogenesis. We generated an N-cadherin-GFP fu- 

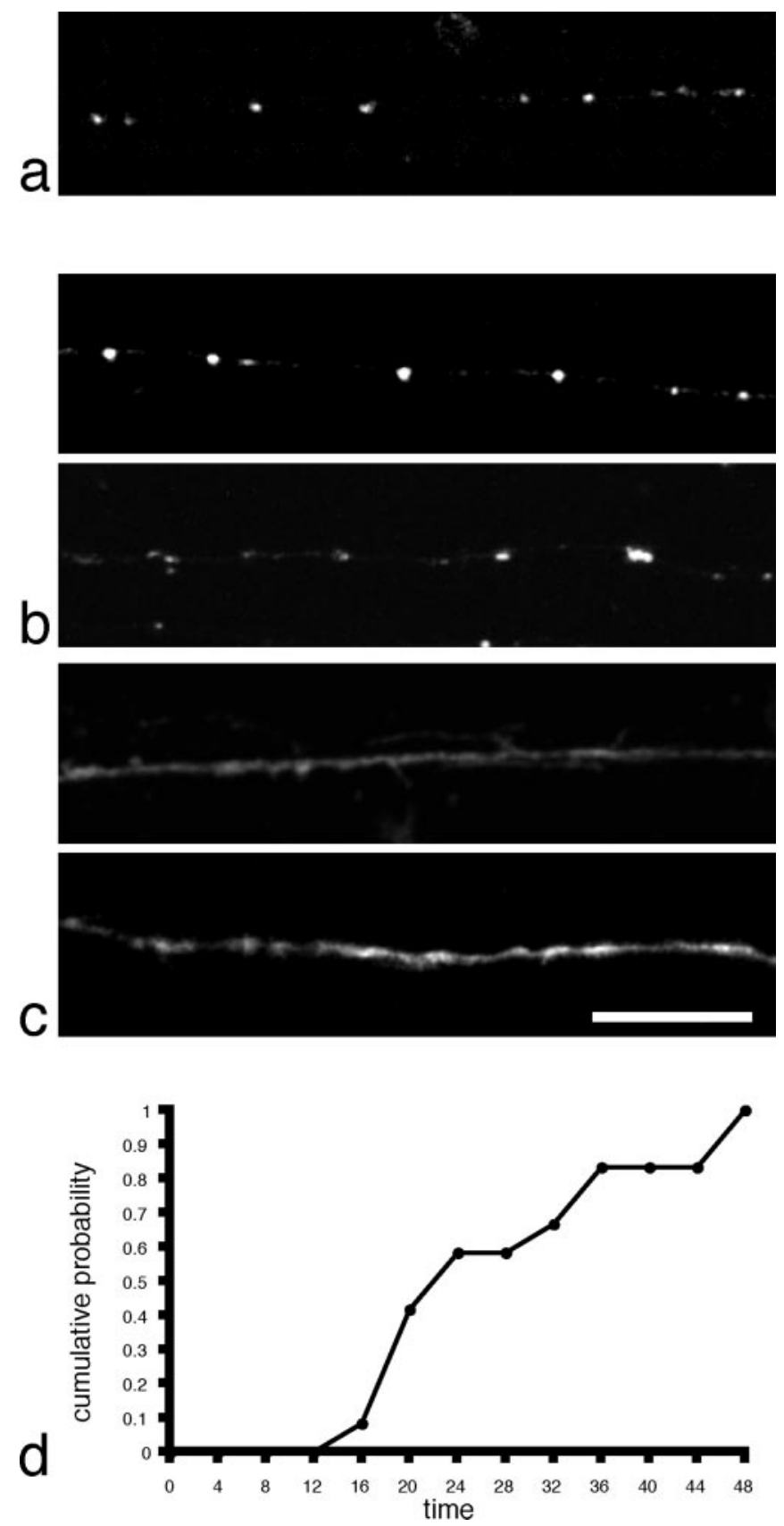

Figure 7. Localization of $\mathrm{N}$-cadherin mutants expressed in Rohon-Beard axons. a, Punctate distribution of full-length Ncad-GFP in a Rohon-Beard axon. $b$, Two examples of Rohon-Beard axons labeled with $\mathrm{Ncad} \Delta$ CP-GFP. Like the full-length $\mathrm{N}$-cadherin, the deletion mutant appears as discreet puncta along the axon, with relatively little background labeling of the plasma membrane along the axon shaft. C, Two examples of Rohon-Beard axons expressing the $\mathrm{Ncad} \Delta \mathrm{EC}$-GFP deletion mutant. In contrast to both Ncad-GFP and Ncad $\Delta$ CP-GFP, there is diffuse, uniform labeling of the plasma membrane in the growth cone and axon. In addition, Ncad $\Delta$ EC-GFP does not accumulate in stable, discrete puncta. Scale bar, $15 \mu \mathrm{m}$. $d$, The time course of Ncad $\triangle$ CP-GFP accumulation is similar to that found for both Ncad-GFP and VAMPGFP, although it appears to stabilize slightly more quickly, with an average time-to-formation of $25 \mathrm{~min}$. Scale bar, $10 \mu \mathrm{m}$.

sion and expressed it in Rohon-Beard sensory neurons in the embryonic zebrafish spinal cord to characterize N-cadherin dynamics and synaptic delivery in vivo (Fig. 1). Ncad-GFP is extremely punctate, accumulating at discrete spots along the axons of Rohon-Beard cells and showing relatively little diffuse labeling along the axonal plasma membrane.
The majority of the $\mathrm{N}$-cadherin puncta labeled highly mobile transport organelles (Fig. 3). Previous time-lapse imaging of GFP-labeled synaptic vesicle proteins in cultured neurons has revealed the existence of dynamic transport packets (Nakata et al., 1998; Ahmari et al., 2000). These transport packets have been shown by electron microscopy to be composed primarily of tubulvesicular structures, pleiomorphic vesicles, and large dense core vesicles (Tsukita and Ishikawa, 1980; Ahmari et al., 2000). The protein composition of these organelles is heterogeneous (Okada et al., 1995; Ahmari et al., 2000; Provoda et al., 2000; Zhai et al., 2001), suggesting either the existence of multiple, distinct populations of transport organelles or the sorting of components through multiple rounds of fusion with plasma membrane and recycling, or both. Our data reveal that $\mathrm{N}$-cadherin is localized to at least two distinct forms of transport packet: discrete-punctate (Fig. 3) and tubulovesicular structures (Fig. 4). The predominantly anterograde direction of tubulovesicular traffic suggests that these organelles may be responsible for delivery of material to the axonal growth cone. The discrete-punctate transport packets are highly mobile, moving in both anterograde and retrograde directions. By analogy to the biogenesis of synaptic vesicles, which are believed to form by local recycling at the nerve terminal (Hannah et al., 1999), the discrete-punctate transport packets may be the result of local recycling and retrieval of $\mathrm{N}$-cadherin from the plasma membrane. It is possible that much of the sorting of axonal membrane proteins could occur through endocytosis after fusion of tubulovesicular organelles and membrane addition in the growth cone.

In addition to the mobile transport packets, Rohon-Beard axons contained stable $\mathrm{N}$-cadherin puncta that formed shortly after passage of the growth cone, with an average time-toformation of $32 \mathrm{~min}$. There are a number of reasons to suspect that these puncta are localized at nascent synapses. First, in more mature axons, large Ncad-GFP puncta were shown to be colocalized with the synaptic vesicle marker SV2 (Fig. $2 e-g$ ). Similarly, stable Ncad-YFP puncta were found to colocalize with accumulations of VAMP-CFP (Fig. $2 h-j$ ). Moreover, previous work has suggested that the formation of axonal varicosities, both in vitro (Hatada et al., 1999) and in vivo (Jontes et al., 2000), provides an early morphological marker of nascent presynaptic boutons. The accumulation of stable $\mathrm{N}$-cadherin puncta in the trail of the growth cone is very similar to the formation of axonal varicosities. In addition, we observe that the accumulation of $\mathrm{N}$-cadherin in Rohon-Beard axons occurs with a time course essentially identical to that observed for VAMP-GFP, a common marker of synaptic vesicle clusters (Ahmari et al., 2000; Alsina et al., 2001; Tokuoka et al., 2002). These observations are consistent with the fact that embryos become touch-sensitive within hours of axon extension (Saint-Amant and Drapeau, 1998). Finally, N-cadherin has been widely shown to be a component of developing synapses (Beesley et al., 1995; Benson and Tanaka, 1998; Huntley and Benson, 1999; Zhang and Benson, 2001; Togashi et al., 2002).

Accumulation of $\mathrm{N}$-cadherin at nascent synapses could, in principle, occur by at least two basic mechanisms. Time-lapse observations of E-cadherin-GFP in cultured epithelial cells have shown that E-cadherin clusters appear to form by lateral recruitment from a diffuse pool of molecules present on the plasma membrane (Adams et al., 1998). In contrast, expression of $\mathrm{N}$ cadherin-GFP in fibroblast cells has suggested a highly dynamic process of intracellular trafficking and recycling, in which cellcell contact recruits $\mathrm{N}$-cadherin-positive transport organelles to the plasma membrane (Mary et al., 2002). Our data reveal that Ncad-GFP labels highly mobile transport organelles similar to 
those observed in fibroblasts. The low level of plasma membrane staining of Ncad-GFP labeled Rohon-Beard neurons and the extensive trafficking of Ncad-GFP suggests that delivery of $\mathrm{N}$-cadherin to nascent synapses occurs through recruitment of transport organelles to sites of cell-cell contact, as was observed in fibroblasts, rather than recruitment from a diffuse plasma membrane pool. It is worth noting that the delivery of $\mathrm{N}$-cadherin to synapses in discrete organelles and the recycling of $\mathrm{N}$-cadherin by internalization represents an efficient way of both targeting $\mathrm{N}$-cadherin to new sites of cell contact and of rapidly destabilizing synapses for disassembly and elimination.

A hallmark of the classical cadherins is their highly conserved cytoplasmic domain, which links them to the actin cytoskeleton through the formation of a 1:1:1 stoichiometric complex with $\beta$-catenin and $\alpha$-catenin (Nagafuchi and Takeichi, 1989; Ozawa et al., 1989). The $\beta$-catenin binding site of E-cadherin has been shown to be important not only for cell adhesion, but also export from the endoplasmic reticulum and targeting to the cell surface (Chen et al., 1999). Surprisingly, Ncad $\Delta$ CP_GFP is transported within axons and accumulates at nascent synaptic sites in a manner similar to that of full-length Ncad-GFP. It is possible that the proper targeting of Ncad $\Delta \mathrm{CP}-\mathrm{GFP}$ to nascent synapses is caused by the formation of endogenous $\mathrm{N}$-cadherin:Ncad $\Delta \mathrm{CP}-\mathrm{GFP}$ hetero-cis-dimers. Thus, rather than being an $\mathrm{N}$-cadherinspecific dominant-negative, $\mathrm{Ncad} \Delta \mathrm{CP}-\mathrm{GFP}$ may represent a relatively noninterfering label of $\mathrm{N}$-cadherin dimers. It appears that for N-cadherin, in Rohon-Beard neurons, an intact cytoplasmic domain and $\beta$-catenin interaction do not appear to be necessary for export from the endoplasmic reticulum (Chen et al., 1999), indicating a substantial mechanistic difference between E-cadherin and $\mathrm{N}$-cadherin that is caused by either an intrinsic difference between the two proteins or a dependence on cell type. This difference between classical cadherins is striking in light of the high degree of conservation in the cytoplasmic domain. In contrast, Ncad $\Delta$ EC-GFP is not appropriately restricted to transport organelles. Because this mutant probably does not form cisdimers, but would otherwise appear wild-type from the cytoplasm, our data suggest that the extracellular domain and possibly cis-dimerization are important for appropriate localization of $\mathrm{N}$-cadherin. This could be caused by a misdirected delivery to the plasma membrane through a constitutive pathway, or through a deficiency in Ncad $\Delta$ EC-GFP endocytic retrieval from the plasma membrane. Recently, the role of cadherin trafficking and recycling in the formation of adhesive contacts has been emphasized, with p120 catenin being implicated as an important regulator of cadherin delivery to the cell surface (Chen et al., 2003; Davis et al., 2003; Xiao et al., 2003). Deletion mutants similar to Ncad $\Delta \mathrm{EC}$-GFP have been shown to act as pan-cadherin dominant negatives in other systems, having effects on adhesiveness (Fujimori and Takeichi, 1993), neurite outgrowth (Riehl et al., 1996), and synaptogenesis (Togashi et al., 2002). However, in vivo, Ncad $\Delta$ EC-GFP has no obvious effects on axon initiation or extension in Rohon-Beard neurons. It will be interesting to determine any downstream effects Ncad $\Delta$ EC-GFP might have on synaptogenesis and synaptic organization.

In this paper, we have provided the first in vivo observations of synaptic adhesion molecule trafficking. We demonstrate that expression of Ncad-GFP in developing axons labels two distinct types of transport organelle: discrete-punctate and tubulovesicular. Our data also suggest that $\mathrm{N}$-cadherin is a very early component of developing synapses, being delivered with a time course similar to that for synaptic vesicle precursors. Finally, expression of $\mathrm{N}$-cadherin deletion mutants reveals differential effects on lo- calization, demonstrating the feasibility of performing detailed cell biological studies in the living organism.

\section{References}

Adams CL, Chen YT, Smith SJ, Nelson WJ (1998) Mechanisms of epithelial cell-cell adhesion and cell compaction revealed by high-resolution tracking of E-cadherin-green fluorescent protein. J Cell Biol 141:1105-1119.

Ahmari SE, Buchanan J, Smith SJ (2000) Assembly of presynaptic active zones from cytoplasmic transport packets. Nat Neurosci 3:445-451.

Alsina B, Vu T, Cohen-Cory S (2001) Visualizing synapse formation in arborizing optic axons in vivo: dynamics and modulation by BDNF. Nat Neurosci 4:1093-1101.

Bamji SX, Shimazu K, Kimes N, Huelsken J, Birchmeier W, Lu B, Reichardt LF (2003) Role of $\beta$-catenin in synaptic vesicle localization and presynaptic assembly. Neuron 40:719-731.

Beesley PW, Mummery R, Tibaldi J (1995) N-cadherin is a major glycoprotein component of isolated rat forebrain postsynaptic densities. J Neurochem 64:2288-2294.

Benson DL, Tanaka H (1998) N-cadherin redistribution during synaptogenesis in hippocampal neurons. J Neurosci 18:6892-6904.

Bozdagi O, Shan W, Tanaka H, Benson DL, Huntley GW (2000) Increasing numbers of synaptic puncta during late-phase LTP: N-cadherin is synthesized, recruited to synaptic sites, and required for potentiation. Neuron 28:245-259.

Chen X, Kojima S-I, Borisy GG, Green KJ (2003) p120 catenin associates with kinesin and facilitates the transport of cadherin-catenin complexes to intercellular junctions. J Cell Biol 163:547-557.

Chen YT, Stewart DB, Nelson WJ (1999) Coupling assembly of the E-cadherin/beta-catenin complex to efficient endoplasmic reticulum exit and basal-lateral membrane targeting of E-cadherin in polarized MDCK cells. J Cell Biol 144:687-699.

Davis MA, Ireton RC, Reynolds AB (2003) A core function for p120-catenin in cadherin turnover. J Cell Biol 163:525-534.

Fannon AM, Colman DR (1996) A model for central synaptic junctional complex formation based on the differential adhesive specificities of the cadherins. Neuron 17:423-434.

Fujimori T, Takeichi M (1993) Disruption of epithelial cell-cell adhesion by exogenous expression of a mutated nonfunctional $\mathrm{N}$-cadherin. Mol Biol Cell 4:37-47.

Hannah MJ, Schmidt AA, Huttner WB (1999) Synaptic vesicle biogenesis. Annu Rev Cell Dev Biol 15:733-798.

Hatada Y, Wu F, Silverman R, Schacher S, Goldberg DJ (1999) En passant synaptic varicosities form directly from growth cones by transient cessation of growth cone advance but not of actin-based motility. J Neurobiol 41:242-251.

Higashijima S, Hotta Y, Okamoto H (2000) Visualization of cranial motor neurons in live transgenic zebrafish expressing green fluorescent protein under the control of the islet-1 promoter/enhancer. J Neurosci 20:206-218.

Hirokawa N (1998) Kinesin and dynein superfamily proteins and the mechanism of organelle transport. Science 279:519-526.

Huntley GW, Benson DL (1999) Neural (N)-cadherin at developing thalamocortical synapses provides an adhesion mechanism for the formation of somatopically organized connections. J Comp Neurol 407:453-471.

Jontes JD, Buchanan J, Smith SJ (2000) Growth cone and dendrite dynamics in zebrafish embryos: early events in synaptogenesis imaged in vivo. Nat Neurosci 3:231-237.

Kemler R (1992) Classical cadherins. Semin Cell Biol 3:149-155.

Köster RW, Fraser SE (2001) Tracing transgene expression in living zebrafish embryos. Dev Biol 233:329-346.

Kraszewski K, Mundigl O, Daniell L, Verderio C, Matteoli M, De Camilli P (1995) Synaptic vesicle dynamics in living cultured hippocampal neurons visualized with CY3-conjugated antibodies directed against the lumenal domain of synaptotagmin. J Neurosci 15:4328-4342.

Lele Z, Folchert A, Concha M, Rauch GJ, Geisler R, Rosa F, Wilson SW, Hammerschmidt M, Bally-Cuif L (2002) parachute/n-cadherin is required for morphogenesis and maintained integrity of the zebrafish neural tube. Development 129:3281-3294.

Mary S, Charrasse S, Meriane M, Comunale F, Travo P, Blangy A, GautierRouviere C (2002) Biogenesis of N-cadherin-dependent cell-cell contacts in living fibroblasts is a microtubule-dependent kinesin-driven mechanism. Mol Biol Cell 13:285-301. 
Nagafuchi A, Takeichi M (1989) Transmembrane control of cadherinmediated cell adhesion: a $94 \mathrm{kDa}$ protein functionally associated with a specific region of the cytoplasmic domain of E-cadherin. Cell Regul $1: 37-44$.

Nakata T, Terada S, Hirokawa N (1998) Visualization of the dynamics of synaptic vesicle and plasma membrane proteins in living axons. J Cell Biol 140:659-674

Niell CM, Meyer MP, Smith SJ (2004) In vivo imaging of synapse formation on a growing dendritic arbor. Nat Neurosci 7:254-260.

Okada Y, Yamazaki H, Sekine-Aizawa Y, Hirokawa N (1995) The neuronspecific kinesin superfamily protein KIF1A is a unique monomeric motor for anterograde axonal transport of synaptic vesicle precursors. Cell 81:769-780.

Ozawa M, Baribault H, Kemler R (1989) The cytoplasmic domain of the cell adhesion molecule uvomorulin associates with three independent proteins structurally related in different species. EMBO J 8:1711-1717.

Phillips GR, Huang JK, Wang Y, Tanaka H, Shapiro L, Zhang W, Shan WS, Arndt K, Frank M, Gordon RE, Gawinowicz MA, Zhao Y, Colman DR (2001) The presynaptic particle web: ultrastructure, composition, dissolution, and reconstitution. Neuron 32:63-77.

Provoda CJ, Waring MT, Buckley KM (2000) Evidence for a primary endocytic vesicle involved in synaptic vesicle biogenesis. J Biol Chem 275:7004-7012.

Riehl R, Johnson K, Bradley R, Grunwald GB, Cornel E, Lilienbaum A, Holt CE (1996) Cadherin function is required for axon outgrowth in retinal ganglion cells in vivo. Neuron 17:837-848.

Saint-Amant L, Drapeau P (1998) Time course of the development of motor behaviors in the zebrafish embryo. J Neurobiol 37:622-632.

Shapira M, Zhai RG, Dresbach T, Bresler T, Torres VI, Gundelfinger ED, Ziv NE, Garner CC (2003) Unitary assembly of presynaptic active zones from Piccolo-Bassoon transport vesicles. Neuron 38:237-252.

Takeichi M (1990) Cadherins: a molecular family important in selective cell-cell adhesion. Annu Rev Biochem 59:237-252.
Tanaka H, Shan W, Phillips GR, Arndt K, Bozdagi O, Shapiro L, Huntley GW Benson DL, Colman DR (2000) Molecular modification of N-cadherin in response to synaptic activity. Neuron 25:93-107.

Tang L, Hung CP, Schuman EM (1998) A role for the cadherin family of cell adhesion molecules in hippocampal long-term potentiation. Neuron 20:1165-1175.

Togashi H, Abe K, Mizoguchi A, Takaoka K, Chisaka O, Takeichi M (2002) Cadherin regulates dendritic spine morphogenesis. Neuron 35:77-89.

Tokuoka H, Yoshida T, Matsuda N, Mishina M (2002) Regulation by glycogen synthase kinase-3beta of the arborization field and maturation of retinotectal projection in zebrafish. J Neurosci 22:10324-10332.

Tsukita S, Ishikawa H (1980) The movement of membranous organelles in axons. Electron microscopic identification of anterogradely and retrogradely transported organelles. J Cell Biol 84:513-530.

Uchida N, Honjo Y, Johnson KR, Wheelock MJ, Takeichi M (1996) The catenin/cadherin adhesion system is localized in synaptic junctions bordering transmitter release zones. J Cell Biol 135:767-779.

Xia Z, Dudek H, Miranti CK, Greenberg ME (1996) Calcium influx via the NMDA receptor induces immediate early gene transcription by a MAP kinase/ERK-dependent mechanism. J Neurosci 16:5425-5436.

Xiao K, Allison DF, Buckley KM, Kottke MD, Vincent PA, Faundez V, Kowalczyk AP (2003) Cellular levels of p120 catenin function as a set point for cadherin expression levels in microvascular endothelial cells. J Cell Biol 163:535-545.

Yamamoto A, Amacher SL, Kim SH, Geissert D, Kimmel CB, De Robertis EM (1998) Zebrafish paraxial protocadherin is a downstream target of spadetail involved in morphogenesis of gastrula mesoderm. Development 125:3389-3397.

Zhai RG, Vardinon-Friedman H, Cases-Langhoff C, Becker B, Gundelfinger ED, Ziv NE, Garner CC (2001) Assembling the presynaptic active zone: a characterization of an active one precursor vesicle. Neuron 29:131-143.

Zhang W, Benson DL (2001) Stages of synapse development defined by dependence on F-actin. J Neurosci 21:5169-5181. 